\title{
Removing Bowtie Phenomenon by Correction of Panoramic Effect in MODIS Imagery
}

\author{
A.H.Souri \\ Department of Surveying and Geomatic Engineering, \\ College of Engineering, University of Tehran, Iran
}

\author{
A.Azizi \\ Department of Surveying and Geomatic Engineering, \\ College of Engineering, University of Tehran, Iran
}

\begin{abstract}
The angular Instantaneous Field Of View (IFOV) of rotating mirror is constant in flying spot scanners and as a result the more area is viewed by mirror with going out of nadir condition and it will cause gradually compression of the effective pixel size at the extremities of scan line. This error is known as panoramic error that is so more sensible in sensors which have high angular Field Of View (FOV). Moderate Resolution Imaging Spectroradiometer (MODIS) sensorwith high FOV $\left(110^{\circ}\right)$ has been complicated by the so-called bowtie effect. The source of error is panoramic distortion that is exacerbated by Earth curvature. This effect causes an overlap of the satellite scan lines producing a data repetition. Bow-tie prevents reaching both real shape and area of objects especially those that are far from the nadir and can be considered as a great systematic error especially for snow maps, dust and cloud researches. Due to lack of heeding utilization geometric source and mathematical transformations of panoramic distortion, former proposed algorithms for correction of bow-tie phenomenon were unnecessarily complicated. In this paper, a rigorous model is proposed in order to remove MODIS bowtie effect. The model is based on geometry projection of panoramic error with Earth curvature. The proposed method requires no ephemeris information and is established from the parameters of MODIS platform. The validity of the algorithm is verified by a scene including Urmia Lake in Iran. The results retrieved by the method demonstrated high both speed and accuracy.
\end{abstract}

Keywords: Bowtie effect, MODIS, panoramic error.

\section{INTRODUCTION}

The Moderate Resolution Imaging Spectroradiometer (MODIS) is acquiring daily global data in 36 spectral bands from $0.4 \mu \mathrm{m}$ to $14.4 \mu \mathrm{m}$. 29 bands with $1 \mathrm{~km}, 5$ with $500 \mathrm{~m}$, and 2 with $250 \mathrm{~m}$ nadir pixel dimensions [1] MODIS is a whiskbroom electro optical instrument that uses the forward motion of the satellite to prove the along-track direction of scan [2] The electromagnetic radiation reflected or emitted from the Earth is reflected into the rotating two-sided scan mirror [2]. MODIS simultaneously senses, in each band, 10 rows of $1 \mathrm{~km}$ detector pixels, 20 rows of $500 \mathrm{~m}$ detector pixels, and 40 rows of $250 \mathrm{~m}$ detector pixels. Each row corresponds to a single scan line of the sensor that it nominally composed of $13541 \mathrm{~km}, 2708$ $500 \mathrm{~m}$, and $5416250 \mathrm{~m}$ samples [2].We will outline in the next section that the important parameter that exacerbates panoramic error is high FOV (Field Of View) of MODIS which changes from $-55^{\circ}$ to $+55^{\circ}$; in addition the wide swath of MODIS which is approximately $2340 \mathrm{~km}$ gets involve the effect of Earth curvature intensively. Therefore in [3] has been shown that at the scan edge, the projection of a MODIS detector's IFOV (Instantaneous Field Of View) onto the surface is approximately 2.0 and 4.8 times larger than at nadir along track and scan directions, respectively. Richard in [4] explained the source of geometric distortion and correction panoramic effect; he has contented himself by the correction of panoramic with merely $\mathrm{x}$-scale factor and totally has forgotten the distortion along y-direction. Therefore it was necessary to construct precisely the mathematical transformation of this distortion. As a result of panoramic errorwith Earth curvature, MODIS data has been complicated by the so-called "bow-tie effect" which causes an overlap of the satellite scan lines producing a data repetition. This effect increases with the distance from nadir and can be especially dramatic at the edge of the image.Bowtie hinders reaching both shape and area of objects that can be considered as a great systematic error especially for snow maps, snowmelt runoff forecasts and cloud researches. The approaches of removing bow-tie phenomenon were separated to ephemeris and non-ephemeris methods. In ephemeris methods, data is projected to a geographic grid, therefore random and systematic errors including bow-tie will be eliminated at the same time. This method is used in MODIS L1B data processing both by international MODIS/AIRS processing Package and Goddard space flight center (NASA/GSFC). The MODIS swath-to-Grid toolbox is also used this method which is provided by National snow and Ice Data Center [6] Because L1B data products don't have ephemeris parameters and general users cannot use the algorithm mentioned above, a lot of studies have been issued to rectify bow-tie error without need of having ephemeris parameters. An algorithm[7] was developed that read the latitude and longitude files associated with each MODIS scene and included in the hierarchical dataformat (HDF) files provided by NASA Distributed Data Archive Center (DAAC) database. The resulting geocoded image was projected in latitude longitude coordinated and corrected for the bow-tie effect. In[8] the correlation coefficient for the overlapped lines between adjacent scanning strips is calculated; similar work [9] attempted to address bow-tie effect by calculating a regression model that shows the number of overlapping line. MODItools is a black box module developed by the $R \& D$ center that is restricted to run on ENVI software[10]. An algorithm in digital photogrammetry is used to find homo-points in adjacent images and in [11] it was used for searching the overlapped lines between adjacent scanning strips and removing bow-tie effect. The performance was disappointing and this was as a result of heavy calculating task and low efficiency. The latest approach was proposed in [12]; a second order polynomial was created with correlation coefficientmethod by the experiments in China, United States and Australia with 500m resolution. This generic model showed the overlapped line and applied to the whole image, thus an image with no overlapped lines was generated.The study mainly focused on the MODIS L1B data with a spatial resolution of $500 \mathrm{~m}$. Therefore for using $250 \mathrm{~m}$ and $1 \mathrm{~km}$ resolution band the parameters must beadjusted and there is still considerable ambiguity with regard to the model that must begenerated for each image, therefore it would not be applied for all images.Despite these interests, no one to the best 
of our knowledge has approached the problem of bow-tie through rigorous and direct way.

In this paper a rigorous model based on geometry of panoramic error with Earth curvaturewas proposed for removing thebowtie effect. The proposed method required no ephemeris information and wasestablished from the parameters of MODIS platform. It could be applied to all MODIS imagery with fixed and accurate parameters. This paper divided into 5 sections; section two describes the concepts and geometric transformation of panoramic error with earth curvature. In section three the case study is introduced. Section four introduces details of the proposed method. Section five describes the results of the method in case study and final section remarks the major conclusion drawn from this research and gives discussion of future directions.

\section{THEORY}

\subsection{Panoramic Error}

For flying spot scanners the angular IFOV is constant. As a result the more area is viewed by mirror with going out of nadir condition and it will cause gradually compression of the effective pixel size at the extremities of scan line. An exaggerated instance for realizing this effect is that a huge circle covered the whole scene of our image on Earth will be captured as vertical ellipse. This effect is known as panoramic error or tangent distortion and is shown for MODIS imagery in figure1; note that objects that are far from nadir has been squeezed.

There is another perspective for dealing with the error; the sensor with fixed IFOV stores a plane (Earth without curvature) from specific height called " $h$ " in cylinder with IFOV $\times \mathrm{h}$ radius. In fact the obtained image is projection of a plane into cylinder. Accordingly following transformation can be regarded as this map projection.

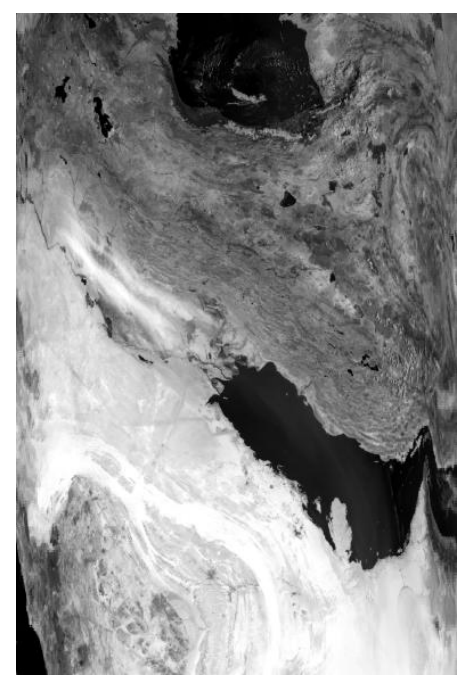

Fig. 1. Panoramic error in a MODIS scene

Assume the IFOV is $\beta$, the pixel dimension at nadir is $p, h$ is altitude and $\theta$ is scan angle. According to figure 2 while the sensor is acquiring data, the cross-section of sampling cone in surface, changes from circle in nadir condition to elliptical shape in further pixels that has $\operatorname{psec}^{2}(\theta)$ and $p \sec (\theta)$ for major and minor axis respectively.

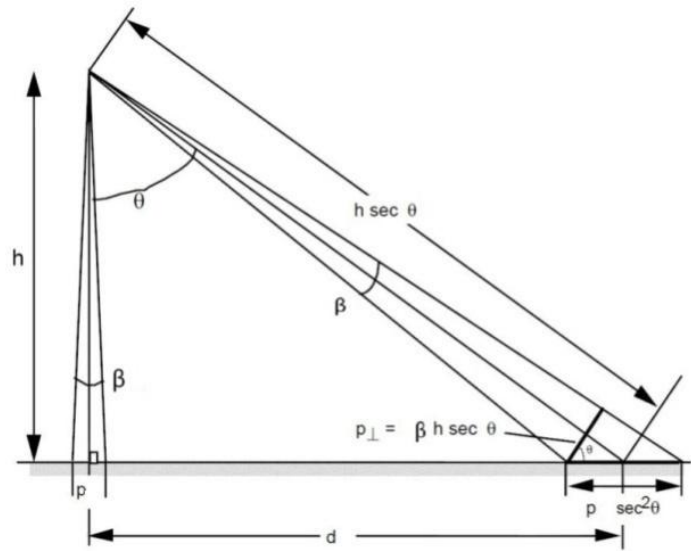

Fig. 2.Panoramic distortion

Therefore this error can be corrected by exerting two different scale factors on input space (cylinder) in order to obtain output space (plane), this transformation is given by:

$$
\begin{gathered}
{\left[\begin{array}{l}
u \\
v
\end{array}\right]_{\text {output }}=p\left[\begin{array}{cc}
\sec ^{2}(\theta) & 0 \\
0 & \sec (\theta)
\end{array}\right] \times\left[\begin{array}{l}
x \\
y
\end{array}\right]_{\text {input }}} \\
\theta=\frac{\mathrm{x} \times \theta_{\max }}{\mathrm{w}}(2) \mathrm{p}=\beta \mathrm{h}
\end{gathered}
$$

where $\mathrm{x}$ and $\mathrm{y}$ are image coordinates in principal point coordinate system. $\theta_{\text {max }}$ is FOV and $\mathrm{W}$ is width of input image. Since $s e c$ is an ascendant function, for higher scan angles the distortion will be more intensive. Another point is non-equivalent of $\mathrm{x}$-scale and $\mathrm{y}$-scale that leads to creation of hyperbola shape.

\subsection{Panoramic error with Earth curvature}

Aircraft scanning systems because of low altitude and some space scanners such as LANDSAT or SPOT because of the narrowness of their swath are not affected by earth curvature. For MODIS with an approximate swath width of $2340 \mathrm{~km}$ and an altitude of $705 \mathrm{~km}$ it can be shown that the deviation of the earth's surface from a plane amounts to less than $1 \%$ over the swath, which seems insignificant [4]. However it is the inclination of the earth's surface over the swath that causes the greater effect [4]. The figure 3 shows Earth curvature intensify panoramic distortion in two ways; firstly, the transformation of $p \perp t o p_{\theta}$ has been increased by adding term of $\varphi$, the angle subtended at the center of the Earth. Secondly, Earth curvature adds extra height to sensor altitude which causes growing size of pixels. The amount of sensor altitude can be formulated by:

$$
h^{\prime}=h+R(1-\cos \varphi)(4)
$$

where $\mathrm{R}$ is Earth radius and $\varphi$ is given by:

$$
\varphi=\sin ^{-1}\left(\frac{R+h}{R} \times \sin \theta\right)-\theta(5)
$$

This effect can be rectified by eq6:

$$
\left[\begin{array}{l}
u \\
v
\end{array}\right]_{\text {output }}=p\left[\begin{array}{cc}
\sec \theta \times \sec (\theta+\varphi) & 0 \\
0 & \sec (\theta)
\end{array}\right] \times\left[\begin{array}{l}
x \\
y
\end{array}\right]_{\text {input }}
$$




$$
\theta=\frac{x \times \theta_{\max }}{W}(7) p=\beta h^{\prime}
$$

Note that eq(6) reduces to eq(1) if $\varphi=0$. With term of $\varphi$ and extra height, exacerbating panoramic effect can be clearly understood.

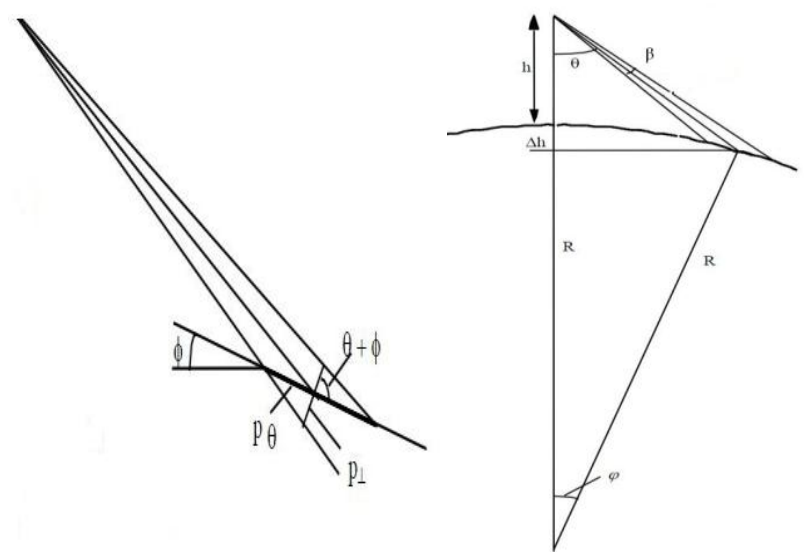

Fig. 3.Panoramic with Earth curvature distortion

\subsection{Bow-tie effect}

As seen in previous section, panoramic error with earth curvature compressed the image in both $\mathrm{x}$ and $\mathrm{y}$ directions. The main point is existence of $y$-scale that leads to overlapping adjacent scans(see figure4). This overlapping is $10 \%$ from nadir that occurring at scan angles of $24^{\circ}$ from nadir and it increases almost to $50 \%$ at the scan edge [5]. Consequently the same point on the Earth's surface may be sensed by more than one consecutive scans at the scan edge. This phenomenon socalled bow-tie is less evident in sensors which have merely one detector but in MODIS with up to 40 detectors it is intensively discernable. Figure5 represents bow-tie in Urmia Lake; note this phenomenon prevents obtaining real shape and area.

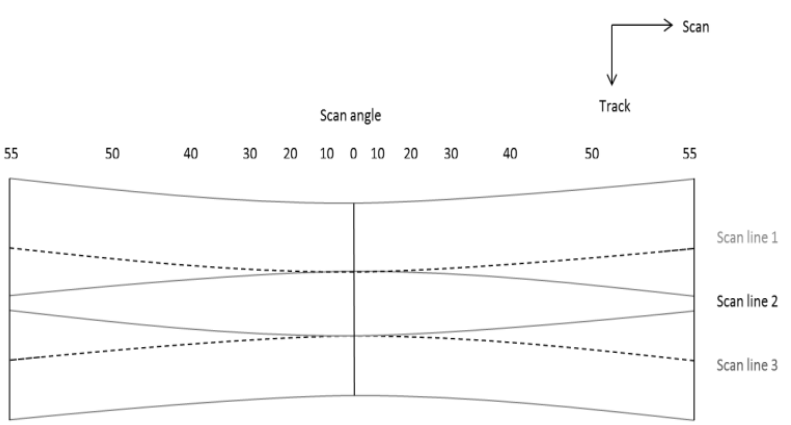

Fig. 4.Ground projection of three consecutive MODIS scans showing the scan geometry and the bow-tie effect.

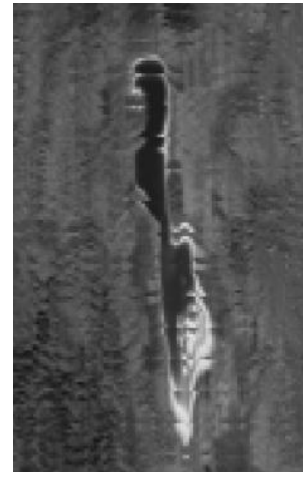

Fig. 5.Bow-tie effect in Urmia Lake

\section{METHODOLOGY}

The proposed method in this research was based on correction of panoramic distortion with earth curvature. Firstly, the rows of distorted image which was in cylinder space must be divided into the number of detectors. For instance,the 26th band of MODIS had ten detectors and 2030*1354 image size. Therefore it was divided into $10 * 1354$ segments.Afterwards equation (6) exerted on these segments in order to rectify panoramic error with Earth curvature distortion. Since no distortion could be found in nadir condition $(\theta=0)$, transformed segments could be adjoined in nadir consecutively. Accordingly, repeated points overlapped and as a result bowtie phenomenon was eliminated. The overview of the methodology is shown in figure6.

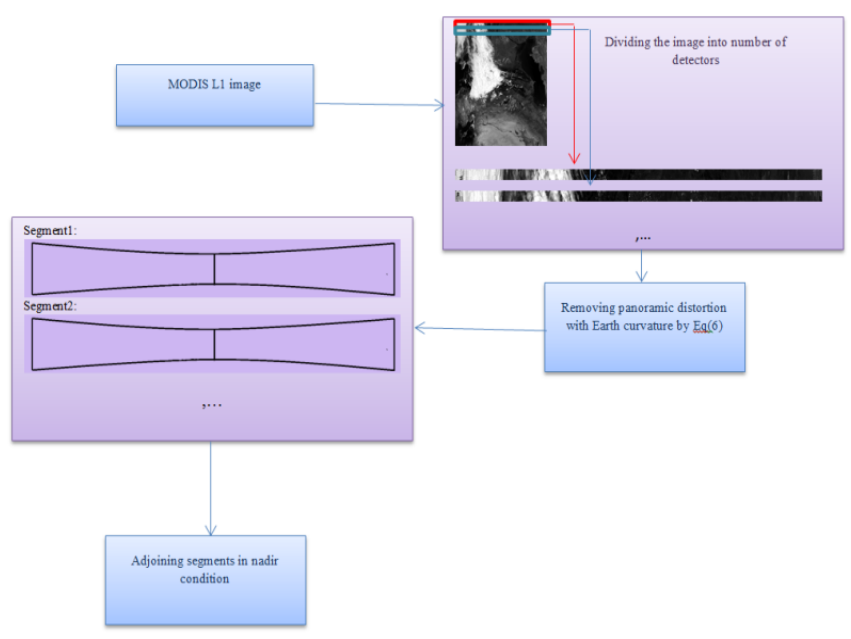

Fig.6. The flowchart of the proposed method.

\section{CASE STUDY}

In order to implement the proposed method, 19th band of MODIS that is appropriate for water vapor retrieval, from Terra spacecraft was used. The location of this case is Iran. The band had ten detectors and 2030*1354 image size (see figure7). All the MODIS products are available for free download at (MODIS data website) in HDF format. 


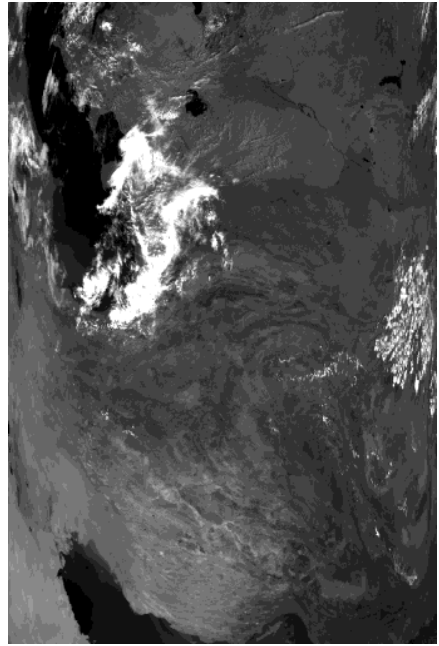

Fig. 7.Case study.

\section{RESULT AND DISCUSSION}

Since the important variable parameter in the model was sensor altitude, it was necessary to determine how sensitive eq (6) was to changes in the value of the sensor altitude.Sensitivity may then be measured by monitoring changes in the output which means by partial derivatives. Suppose $f$ and $g$ are partial derivatives of equation (6) in $x$ and y directions respectively:

$$
\begin{aligned}
& \frac{\partial s_{x}}{\partial h}=f(h, R, \theta) \\
& \frac{\partial s_{y}}{\partial h}=g(h, R, \theta)
\end{aligned}
$$

Afterwards, $f$ and $g$ were calculated which are listed in table1.

Table1. Values of partial derivatives

\begin{tabular}{|c|c|}
\cline { 2 - 2 } \multicolumn{1}{c|}{} & Value \\
\hline$f(h=705 \mathrm{~km}, R=6367 \mathrm{~km}, \theta=0)$ & 0.0014 \\
\hline$g(h=705 \mathrm{~km}, R=6367 \mathrm{~km}, \theta=0)$ & 0.0014 \\
\hline$f\left(h=705 \mathrm{~km}, R=6367 \mathrm{~km}, \theta=55^{\circ}\right)$ & 0.0114 \\
\hline$g\left(h=705 \mathrm{~km}, R=6367 \mathrm{~km}, \theta=55^{\circ}\right)$ & 0.0034 \\
\hline
\end{tabular}

The results from table1 describe the error originated from sensor altitude variability for $\mathrm{x}$ and $\mathrm{y}$ directions in the farthest pixel from nadir, are 8.14, 2.43 times more than nadir condition respectively.According to sensitivity analysis, if sensor altitude changed $10 \mathrm{~km}$, the transformation in scan edgewould have 114 and 34 meters error in $\mathrm{x}$ and $\mathrm{y}$ directions respectively. This error in comparison with spatial resolution of MODIS sensor is insignificant. Consequently the sensor altitude was set $705 \mathrm{~km}$ in this work.

The values of used parameters in the eq (6) are listed in table2.
Table2. Used parameters for the transformation

\begin{tabular}{|c|c|}
\hline $\mathrm{H}$ & $705 \mathrm{~km}$ \\
\hline $\mathrm{R}$ & $6367 \mathrm{~km}$ \\
\hline$\theta_{\max }$ & $110^{\circ}$ \\
\hline $\mathrm{p}$ & $1 \mathrm{~km}$ \\
\hline$\beta$ & $0.081241^{\circ}$ \\
\hline$\varphi_{\max }$ & $10.485^{\circ}$ \\
\hline $\mathrm{W}$ & 1354 \\
\hline
\end{tabular}

After exerting equation (6) on segments, transformed pixel was resampled by bi-cubic interpolation which was associated with the sinc function as the optimum interpolation kernel.In the extremities of scan line, $44 \%$ of segments overlapped. The maximum values for scale factors were 4.816 and 2.003 in along and track scan directions respectively. These values correlated satisfactorily with [3]. In figure 8 the panoramic error, earth curvature and bow-tie phenomenon have been eliminated from the image:

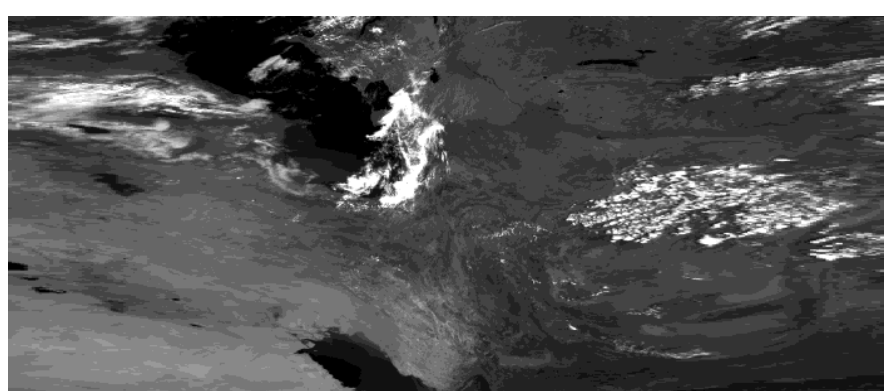

Fig. 8.Rectified image.

Rectified image must be corrected by other systematic and random errors and be projected to a standard map projection;thereforeestimation the accuracy of the result is difficult with human eye's interpretation.Consequently we resort to subset spatially around Urmia Lake region; afterwards the cropped image has been georeferenced with first order polynomial by 5 tie points selected manually fromgeoreferenced L1B MODIS product.Again, uncorrectedL1 image has been georeferenced in same way.The figure9 represents the cropped image with and without performing the proposed method. The difference between sizesof two images is because of selecting manually tie points and this action has been done merely for making the comparison of the result easier. The final result in Urmia Lake is depicted in figure 10. 


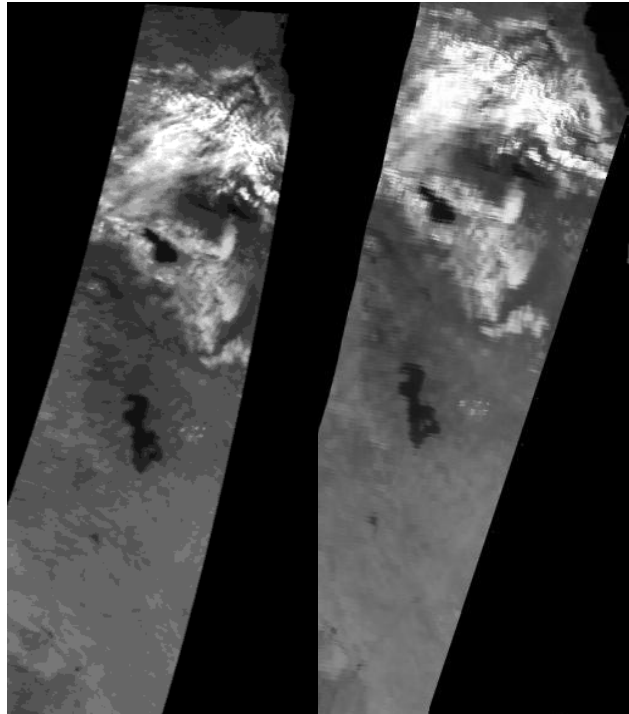

Fig. 9. Left and right images are georefrenced image with and without carrying out the proposed method respectively.

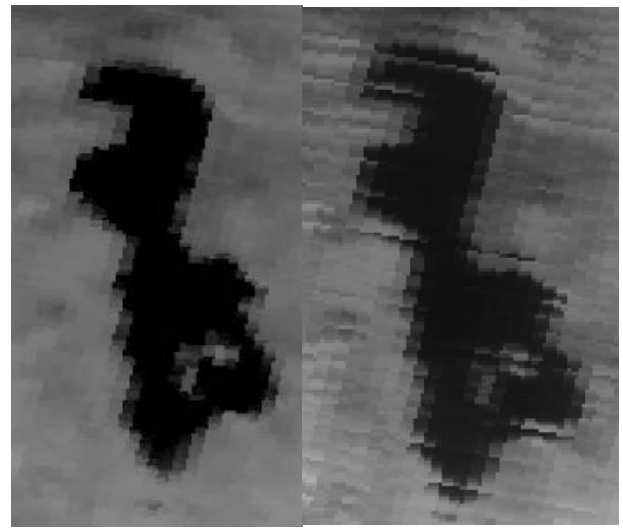

Fig. 10. Left and right images are Urmia Lake with and without carrying out the proposed method respectively.

Acceding to the results, proposed method in this work provedjust how remarkable the rigorous model could rectify panoramic distortion, Earth curvature and bow-tie phenomenon in the same time. The technique showed a clear advantage over former works. It did not demand any ephemeris parameters and also there was no need to create a generic model for each image in order to remove bow-tie effect. This method can be applied to all MODIS images.

\section{CONCLUSION}

In this paper a reliable and accurate transformationfor removing bow-tie effect in MODIS imagery was introduced. It was based on geometric source of panoramic error with Earth curvature and there was no need of knowing ephemeris parameters. It could be applied to each band with different spatial resolution.The method was tested on a scene including Urmia Lake.The results demonstrated high accuracy and performance and this was because of heeding rigorous form that addresses source of the error.Therefore with utilization the proposed transformation, we can hinder having erroneous result especially for snow map cover and cloud researches. Our study provided additional support for calibration wide close- range cameras. With eq(1) one of the systematic errors will be rectified. In addition the mentioned transformation could be useful for creation of panorama images. The proposed approach for removing bow-tie effect could be developed with further studies including: 1)Removing bow-tie phenomenon with an application to cloud researches, 2) Calibration the wide closerange cameras. 3) Creating automatic panorama images with sequence of camera images.

\section{REFERENCES}

[1] Salomonson, V. V., Barnes, W. L., Maymon, P. W., Montgomery, H. E\& ,.Ostrow, H., 1989, MODIS: advanced facility instrument for studies ofthe earth as a system,IEEE Transactions on Geoscience and RemoteSensing, 27, pp. 145-153.

[2] Robert E. Wolfe, Masahiro Nishihama , Albert J. Fleig, James A. Kuyper ,David P. Roy, James C. Storey , Fred S. Patt ,2002, Achieving sub-pixel geolocation accuracy in support of MODIS land science, Remote Sensing of Environment,83, pp. 31-49.

[3] Wolfe, R. E., Roy, D. P., \&Vermote, E., 1998,MODIS land data storage,gridding and compositing methodology: Level 2 Grid, IEEE Transactions on Geoscience and Remote Sensing,36, pp. 1324- 1338.

[4] John, A.Richards, Xiuping, Jia., 2006,Remote Sensing Digital Image Analysis, (4 ${ }^{\text {th }}$ ed.) New York: SpringerVerlag Berlin Heidelberg,pp.55.

[5] Schowengerdt, R. A., 1997, Remote sensing models and methods for image processing", ( $2^{\text {nd }}$ ed.), San Diego: Academic Press,pp. 100-109.

[6] National Snow and Ice Data Center, 2003,MS2GT: The MODIS Swath-to- Grid Toolbox, Available from: http://nsidc.org/data/modis/ms2gt/

[7] Gomez-Landesa, E., Rango, A., Bleiweiss, M., 2004, An algorithm to address the MODIS bowtie effect, Canadian Journal of Remote Sensing, 30(4), 644-650.

[8] XuMeng, Yu Fan., 2005, The Method of eliminating the Bowtie effect in EOS/MODIS 1B Data,ScientiaMeteorologica SINICA,25(3),pp. 257-264.

[9] GuoGuangmeng, 2003,Eliminating Bowtie effect of MODIS Data without Ephemeris, Remote Sensing Technology and Application, 18(3), pp. 172-175.

[10] R\&D center ScanEx, 2004,MODIStools for ENVI, Available from:http://eostation.scanex.ru/software.html

[11] Yu Junhui, Zhang Wanchang,2004, A Method Derived from Digital Photogrammetry used Eliminated Bowtie effect in MODIS L1B Data,Application Research of computers,21 (7),pp.239-240.

[12] Xiongfei Wen, A new prompt algorithm for removing the bowtie effect of MODIS L1b data, The International Archives of the Photogrammetry, Remote Sensing and Spatial Information Sciences, Beijing 2008, Vol. XXXVII. Part B3b.

\section{[13] MODIS}

data,Availablefrom:http://ladsweb.nascom.nasa.gov/data/s earch.html 